\title{
A Deep Residual U-Net Algorithm for Automatic Detection and Quantification of Ascites on Abdominopelvic Computed Tomography Images Acquired in the Emergency Department: Model Development and Validation
}

Hoon $\mathrm{Ko}^{1^{*}}$, MSc; Jimi $\mathrm{Huh}^{2 *}$, MD, PhD; Kyung Won Kim ${ }^{3,4}, \mathrm{MD}, \mathrm{PhD}$; Heewon Chung ${ }^{1}$, MSc; Yousun $\mathrm{Ko}^{5}, \mathrm{PhD}$; Jai Keun $\mathrm{Kim}^{2}$, MD, PhD; Jei Hee Lee ${ }^{2}, \mathrm{MD}, \mathrm{PhD}$; Jinseok Lee ${ }^{1}, \mathrm{PhD}$

\footnotetext{
${ }^{1}$ Department of Biomedical Engineering, Kyung Hee University, Yongin-si, Republic of Korea

${ }^{2}$ The Department of Radiology, Ajou University School of Medicine, Suwon, Republic of Korea

${ }^{3}$ Department of Radiology, Asan Medical Center, University of Ulsan College of Medicine, Seoul, Republic of Korea

${ }^{4}$ Research Institute of Radiology, Asan Medical Center, University of Ulsan College of Medicine, Seoul, Republic of Korea

${ }^{5}$ Biomedical Research Center, Asan Institute for Life Sciences, Asan Medical Center, Seoul, Republic of Korea

*these authors contributed equally
}

\section{Corresponding Author:}

Jinseok Lee, $\mathrm{PhD}$

Department of Biomedical Engineering

Kyung Hee University

1732, Deogyeong-daero

Giheung-gu

Yongin-si, 17104

Republic of Korea

Phone: 82312012570

Email: gonasago@khu.ac.kr

\section{Abstract}

Background: Detection and quantification of intra-abdominal free fluid (ie, ascites) on computed tomography (CT) images are essential processes for finding emergent or urgent conditions in patients. In an emergency department, automatic detection and quantification of ascites will be beneficial.

Objective: We aimed to develop an artificial intelligence (AI) algorithm for the automatic detection and quantification of ascites simultaneously using a single deep learning model (DLM).

Methods: We developed 2D DLMs based on deep residual U-Net, U-Net, bidirectional U-Net, and recurrent residual U-Net (R2U-Net) algorithms to segment areas of ascites on abdominopelvic CT images. Based on segmentation results, the DLMs detected ascites by classifying CT images into ascites images and nonascites images. The AI algorithms were trained using 6337 CT images from 160 subjects ( 80 with ascites and 80 without ascites) and tested using 1635 CT images from 40 subjects (20 with ascites and 20 without ascites). The performance of the AI algorithms was evaluated for diagnostic accuracy of ascites detection and for segmentation accuracy of ascites areas. Of these DLMs, we proposed an AI algorithm with the best performance.

Results: The segmentation accuracy was the highest for the deep residual U-Net model with a mean intersection over union (mIoU) value of 0.87 , followed by U-Net, bidirectional U-Net, and R2U-Net models (mIoU values of 0.80, 0.77, and 0.67, respectively). The detection accuracy was the highest for the deep residual U-Net model (0.96), followed by U-Net, bidirectional U-Net, and R2U-Net models $(0.90,0.88$, and 0.82, respectively). The deep residual U-Net model also achieved high sensitivity (0.96) and high specificity (0.96)

Conclusions: We propose a deep residual U-Net-based AI algorithm for automatic detection and quantification of ascites on abdominopelvic CT scans, which provides excellent performance.

(J Med Internet Res 2022;24(1):e34415) doi: $\underline{10.2196 / 34415}$ 


\section{KEYWORDS}

ascites; computed tomography; deep residual U-Net; artificial intelligence

\section{Introduction}

Currently, computed tomography (CT) of the abdomen and pelvis continues to be the primary modality for patients who visit an emergency department for abdominal pain or trauma, especially in time-critical situations [1]. In emergency situations, immediate assessment of $\mathrm{CT}$ is required, but limited radiologic resources may hamper or delay the recognition of patients who need urgent intervention or surgery [2]. To overcome these challenges, the development of artificial intelligence (AI) techniques using a deep learning model (DLM) to detect critical findings on CT images might be a possible solution [3].

On abdominopelvic CT images, several findings indicate emergent or urgent conditions, including ascites (ie, intra-abdominal free fluid), free gas, abscess, and fat stranding [1]. Of these, presence of ascites is a common finding in various acute abdominal diseases and intra-abdominal organ injury [4]. In addition, quantification of ascites is also important, as the amount of free fluid may correlate with the severity of injury [5].

There has been only one study that developed a DLM to detect ascites, but that DLM did not quantify the amount of fluid. That study used a convolutional neural network (CNN) classification algorithm to discriminate $\mathrm{CT}$ images with fluid from CT images without fluid, which achieved $85 \%$ sensitivity and $95 \%$ specificity [3]. In contrast to that study, we attempted to develop an AI segmentation algorithm that can perform both detection of ascites as well as the quantification of the volume of ascites at the same time. A segmentation value of zero means no ascites, and segmentation values of the area of ascites can be used to quantify the exact volume of ascites. In addition, we tried to increase the detection accuracy of the AI algorithm.

Recently, several state-of-the-art DLM algorithms for segmentation of CT images have been proposed, including U-Net [6], bidirectional U-Net [7], recurrent residual U-Net (R2U-Net) [8], and a deep residual U-Net CNN [9]. U-Net is one of the deep learning networks with an encoder-decoder architecture, which employs skip connections to combine low-level feature maps from an encoder and high-level semantic feature maps from a decoder. Since U-Net allows for the use of location and context at the same time, and works well with very few training samples, it has been widely used in medical image segmentation [10-13]. In addition, variant models based on
U-Net, such as bidirectional U-Net, R2U-Net, and a deep residual U-Net, have been applied to medical image segmentation.

Of these, we hypothesized that a deep residual U-Net might be the best algorithm for segmentation because it combines the strengths of residual learning and U-Net. The residual network has several advantages [14-16]. First, it accelerates the speed of training of the deep networks. Second, it requires fewer parameters by increasing the depth of the network instead of widening the network. Third, it reduces the effect of the vanishing gradient problem. Last, it provides high accuracy in network performance, especially in image classification and segmentation. However, no study has been reported that used a deep residual U-Net algorithm for the segmentation of ascites on CT images. Thus, we aimed to develop an optimized deep residual U-Net algorithm to detect and quantify ascites on CT images, along with a performance comparison with other state-of-the-art networks.

\section{Methods}

\section{Patients}

This study was approved by the institutional review board of Ajou University Hospital. Informed consent was waived. From January 1 to March 1, 2020, a total of 1055 patients visited the emergency department and had abdominopelvic CT scans performed. Of these, 205 patients had ascites detected on their CT images. After excluding 5 patients who underwent noncontrast CT only, we included 200 patients as the ascites group. Of the remaining 850 patients without ascites, we chose 200 age- and sex-matched controls using the MatchIt package (version 4.0.0) in R software (version 4.0.2; The R Foundation). From the patients in the ascites group and the control group, we randomly selected 100 patients with ascites and 100 patients without ascites for training and testing AI models.

The clinical characteristics of the patients in the control group and ascites group are summarized in Table 1. In the control group, out of 200 patients, unknown cause of abdominal pain $(n=140,70.0 \%)$ was the most common disease category with normal abdominopelvic CT. In contrast, in the ascites group, out of 200 patients, cancer $(n=42,21.0 \%)$, liver cirrhosis $(n=52$, $26.0 \%)$, blunt trauma $(n=37,18.5 \%)$, and infection $(n=28$, $14.0 \%$ ) were the main causes for emergency department visits. The majority of ascites were identified in the pelvic cavity. 
Table 1. Demographic and clinical data of participants in the control group and ascites group.

\begin{tabular}{|c|c|c|}
\hline Variables & Control group $(n=200)$ & Ascites group $(\mathrm{n}=200)$ \\
\hline \multicolumn{3}{|l|}{ Demographics } \\
\hline \multicolumn{3}{|l|}{ Sex, n (\%) } \\
\hline Female & $92(46.0)$ & $101(50.5)$ \\
\hline Male & $108(54.0)$ & $99(49.5)$ \\
\hline Age in years, mean (SD) & $59.7(13.8)$ & $60.2(15.3)$ \\
\hline \multicolumn{3}{|l|}{ Amount of ascites, $n(\%)$} \\
\hline Large & $0(0)$ & $92(46.0)$ \\
\hline Moderate & $0(0)$ & $47(23.5)$ \\
\hline Small & $0(0)$ & $61(30.5)$ \\
\hline \multicolumn{3}{|l|}{ Disease category, n (\%) } \\
\hline Cancer & $14(7.0)$ & $42(21.0)$ \\
\hline Congestive heart failure & $0(0)$ & $3(1.5)$ \\
\hline Liver cirrhosis & $1(0.5)$ & $51(25.5)$ \\
\hline Acute liver failure & $0(0)$ & $3(1.5)$ \\
\hline Infection & $7(3.5)$ & $28(14.0)$ \\
\hline Blunt trauma & $5(2.5)$ & $37(18.5)$ \\
\hline Postoperative status & $32(16.0)$ & $5(2.5)$ \\
\hline Intestinal obstruction & $1(0.5)$ & $10(5.0)$ \\
\hline Renal failure & $0(0)$ & $10(5.0)$ \\
\hline Unknown cause of abdominal pain & $140(70.0)$ & $11(5.5)$ \\
\hline
\end{tabular}

\section{CT Image Acquisition and Analysis}

All patients underwent abdominopelvic CT scans using multichannel multidetector scanners (Somatom Definition Edge or Somatom Definition AS, Siemens Healthineers). Contrast-enhanced CT scans were obtained with intravenous injections of 100 to $150 \mathrm{~mL}$ of a nonionic contrast medium (Iopamiro 300, Bracco Imaging; Omnipaque 300, GE Healthcare) at a rate of 2.5 to $3 \mathrm{~mL} / \mathrm{s}$. The scan parameters were as follows: beam collimation, $0.75 \mathrm{~mm}$; slice thickness, $5 \mathrm{~mm}$; effective tube current-time charge, 200 to $260 \mathrm{mAs}$; and voltage, 100 to $120 \mathrm{kVp}$. In this study, we used only contrast-enhancement CT images. If there were multiphasic CT images, we chose portal venous phase CT images for AI training and validation.

An expert abdominal radiologist (JH, with 13 years' experience) selected CT slices that demonstrated ascites from the ascites group (2461 images from 100 patients). Then, the radiologist selected corresponding CT slices from the control group (5511 images from 100 patients). The radiologist created segmentation maps of ascites in the selected CT slices using ImageJ software (version 1.53j; National Institutes of Health), which served as ground-truth labels.

\section{Training and Validation Data Set and Augmentation}

Table 2 summarizes the training and testing data sets, which were randomly split with a ratio of 8:2 into a training set and a testing set, respectively, in a stratified fashion. The testing set was used only for an independent test of developed models and was never used for training and internal validation.

The training data set was then further separated for training the model ( $80 \%$ of the training set) and for internal validation $(20 \%$ of the training set). To balance the two groups' images as well as reduce overfitting on training data, we employed image augmentation. We randomly drew the training images and applied them to the random combination of angle rotation between -10 and 10 degrees and vertical and horizontal flip. Finally, a total of 48,874 CT images were augmented: 24,437 images from patients with ascites and 24,437 images from healthy subjects.

Table 2. Summary of training and testing data sets.

\begin{tabular}{|c|c|c|c|c|c|c|}
\hline \multirow[t]{2}{*}{ Group } & \multicolumn{2}{|c|}{ Training data, $\mathrm{n}(\%)$} & \multicolumn{2}{|c|}{ Testing data, n (\%) } & \multicolumn{2}{|l|}{ Total, n (\%) } \\
\hline & Subjects $(n=160)$ & Images $(n=6337)$ & Subjects $(n=40)$ & Images $(n=1635)$ & Subjects $(n=200)$ & Images $(n=7972)$ \\
\hline Ascites & $80(50.0)$ & $1969(31.1)$ & $20(50.0)$ & $492(30.1)$ & $100(50.0)$ & $2461(30.9)$ \\
\hline Control & $80(50.0)$ & 4368 (68.9) & $20(50.0)$ & 1143 (69.9) & $100(50.0)$ & $5511(69.1)$ \\
\hline
\end{tabular}




\section{Preprocessing}

For all of the images in the training and testing data sets, we first set the abdomen window according to the Digital Imaging and Communications in Medicine (DICOM) standard, which is a 400 Hounsfield Unit (HU) window width and a $60 \mathrm{HU}$ window level. Subsequently, we down-sampled the DICOM images as well as masked images from an image size of $512 \times$ 512 pixels to $256 \times 256$ pixels, and we normalized the pixel values to a range between 0 and 1 .

\section{Deep Residual U-Net}

We proposed the model for ascites region segmentation based on a single abdomen CT image using a deep residual U-Net algorithm. Figure 1 shows the architecture of our proposed model, which is comprised of three parts: an encoder, a bridge, and a decoder. In the encoder part, the normalized $256 \times$ 256-pixel image as input is encoded into a denser representation. The decoding part, on the other hand, recovers the ascites region by pixel-wise categorization. The bridge part connects the encoding and decoding parts.

In this study, we used the residual learning approach to facilitate the training of deep neural networks and take advantage of the ascites segmentation performance gain in abdomen CT images. Each residual block consists of two paths. One path is the forward pass through batch normalization, activation, and convolutional layers, which are repeated twice. The other path is the skip connection. The outputs from the two paths are added as a single output. In the encoder part (ie, residual blocks 1-4), the output from the residual block is fed into both a subsequent residual block and one of the residual blocks in the decoder part (ie, residual blocks 6-9). Thus, in the decoder part, the residual block has two inputs: one from the encoder and the other from the previous residual block output. In the bridge part, another residual block (ie, residual block 5) connects the encoding part to the decoding part. In this study, we found that four residual blocks in each of the encoder and decoder parts provided the best performance in ascites segmentation. We describe our numerical results and comparisons in the Results section. For all residual blocks, we used the rectified linear unit activation function.

Table 3 summarizes the hyperparameters of the convolutional layers and the output size in each residual block. The normalized $256 \times 256 \times 3$-pixel image as input was fed into residual block 1 , where we used the two convolutional layers with $323 \times$ 3-pixel kernels and a stride of 1 with zero padding. The activation map with a size of $256 \times 256 \times 32$ pixels from residual block 1 was fed into both residual block 2 and residual block 9. In residual block 2 , we used two convolutional layers with $643 \times 3$-pixel kernels and strides of 2 and 1 with zero padding. The activation map with a size of $128 \times 128 \times 64$ pixels from residual block 2 was fed into both residual block 3 and residual block 8 . In residual block 3 , we used two convolutional layers with $1283 \times 3$-pixel kernels and strides of 2 and 1 with zero padding. The activation map with a size of $64 \times 64 \times 128$ pixels from residual block 3 was fed into both residual block 4 and residual block 7 . In residual block 4 , we used two convolutional layers with $2563 \times 3$-pixel kernels and strides of 2 and 1 with zero padding. The activation map with a size of $32 \times 32 \times 256$ pixels from residual block 4 was fed into residual block 5 , where we used two convolutional layers with $5123 \times 3$-pixel kernels and strides of 2 and 1 with zero padding.

The activation map with a size of $16 \times 16 \times 512$ pixels from residual block 5 was fed into residual block 6 , where the input was first up-sampled to $32 \times 32 \times 512$ pixels. In residual block 6 , we used two convolutional layers with $2563 \times 3$-pixel kernels and a stride of 1 with zero padding. The activation map with a size of $32 \times 32 \times 256$ pixels from residual block 6 was fed into residual block 7 , and it was concatenated with the output from residual block 3 . When the two inputs were concatenated, the output from residual block 6 was up-sampled to match the size. In residual block 7, we used two convolutional layers with 128 $3 \times 3$-pixel kernels and a stride of 1 with zero padding. The activation map with a size of $64 \times 64 \times 128$ pixels from residual block 7 was fed into residual block 8 , and it was up-sampled and concatenated with the output from residual block 2 . In residual block 8 , we used two convolutional layers with $643 \times$ 3 -pixel kernels and a stride of 1 with zero padding. The activation map with a size of $128 \times 128 \times 64$ pixels from residual block 8 was fed into residual block 9 , and it was up-sampled and concatenated with the output from residual block 1 . In residual block 9, we used two convolutional layers with $323 \times$ 3-pixel kernels and a stride of 1 with zero padding.

The activation map with a size of $256 \times 256 \times 32$ pixels was then fed into the convolutional layer with a single $1 \times 1$-pixel kernel and a stride of 1 . The resultant activation map with a size of $256 \times 256 \times 1$ pixels was finally fed into a sigmoid layer, which provided the pixel-wise probability of the presence or absence of ascites. 
Figure 1. The architecture of our proposed model for ascites region segmentation based on a single abdomen computed tomography (CT) image. ReLU: rectified linear unit.

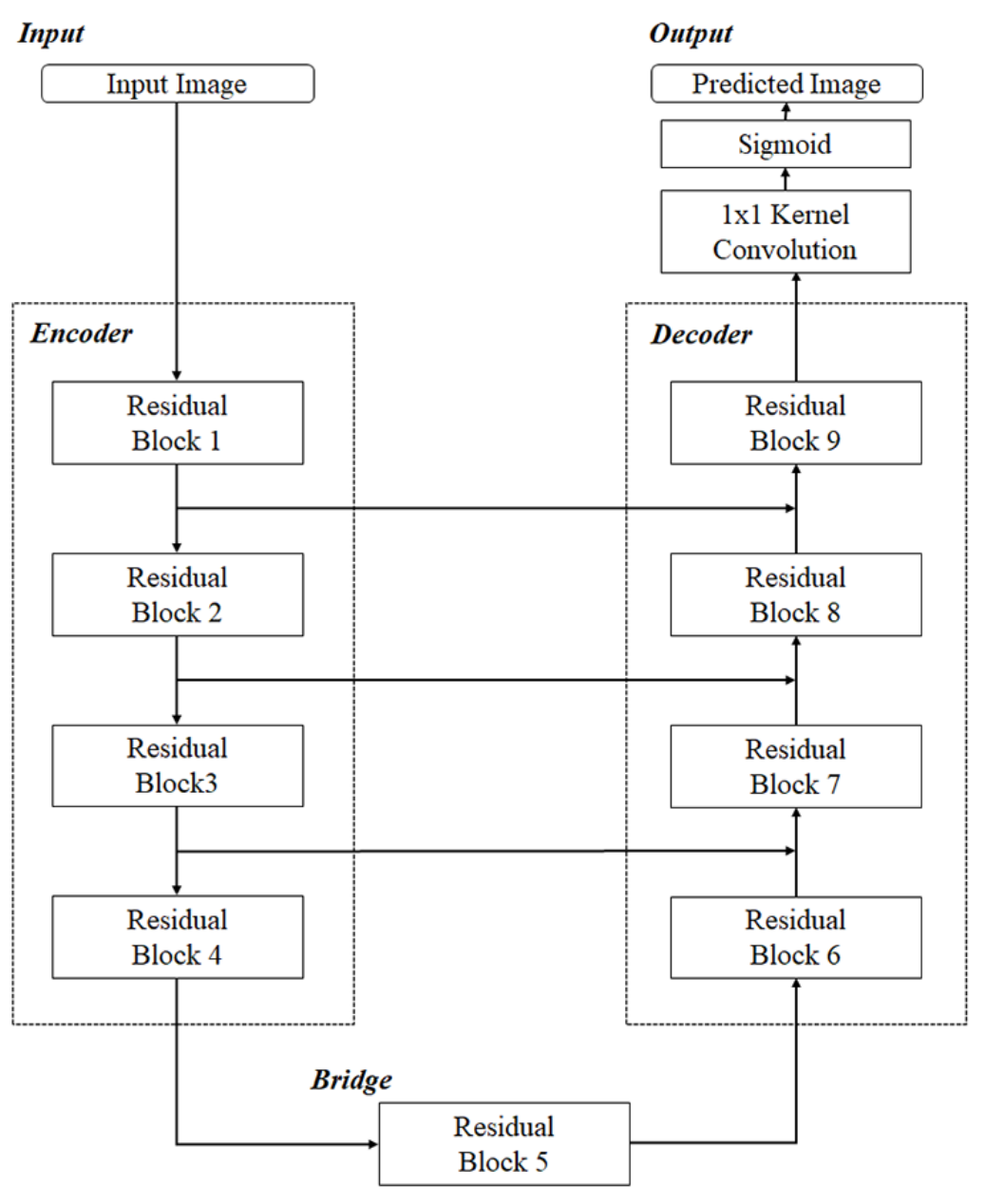

Residual Block

in Encoder and Bridge Part

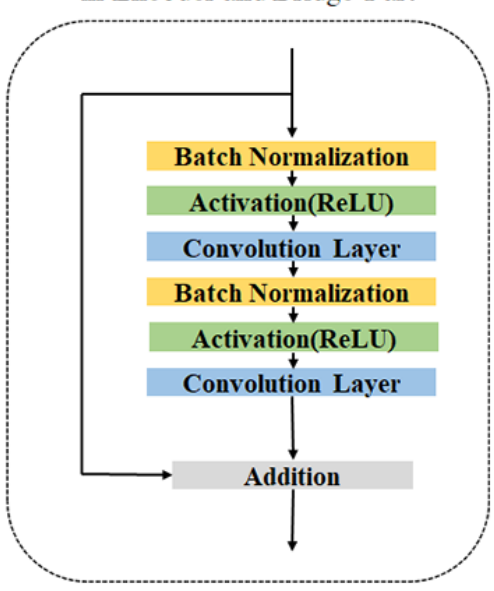

Residual Block in Decoder Part

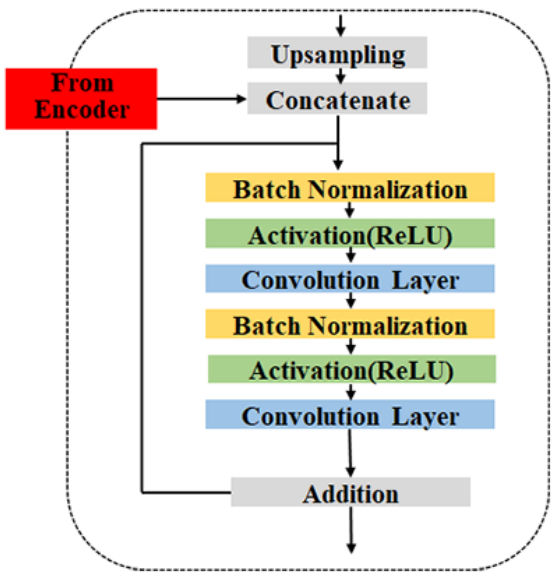


Table 3. Hyperparameters of convolutional layers according to each layer and unit level.

\begin{tabular}{|c|c|c|c|c|}
\hline \multirow[t]{2}{*}{ Model part, unit level, and layer } & \multicolumn{2}{|l|}{ Kernel } & \multirow[t]{2}{*}{ Strides, $\mathrm{n}$} & \multirow[t]{2}{*}{ Output size, pixel } \\
\hline & Filter size, pixels & Filters, $\mathrm{n}$ & & \\
\hline \multicolumn{5}{|l|}{ Input } \\
\hline $\mathrm{N} / \mathrm{A}^{\mathrm{a}}$ & N/A & N/A & N/A & $256 \times 256 \times 3$ \\
\hline \multicolumn{5}{|l|}{ Encoder } \\
\hline \multicolumn{5}{|l|}{ Residual block 1} \\
\hline Convolutional layer 1 & $3 \times 3$ & 32 & 1 & $256 \times 256 \times 32$ \\
\hline Convolutional layer 2 & $3 \times 3$ & 32 & 1 & $256 \times 256 \times 32$ \\
\hline \multicolumn{5}{|l|}{ Residual block 2} \\
\hline Convolutional layer 3 & $3 \times 3$ & 64 & 2 & $128 \times 128 \times 64$ \\
\hline Convolutional layer 4 & $3 \times 3$ & 64 & 1 & $128 \times 128 \times 64$ \\
\hline \multicolumn{5}{|l|}{ Residual block 3} \\
\hline Convolutional layer 5 & $3 \times 3$ & 128 & 2 & $64 \times 64 \times 128$ \\
\hline Convolutional layer 6 & $3 \times 3$ & 128 & 1 & $64 \times 64 \times 128$ \\
\hline \multicolumn{5}{|l|}{ Residual block 4} \\
\hline Convolutional layer 7 & $3 \times 3$ & 256 & 2 & $32 \times 32 \times 256$ \\
\hline Convolutional layer 8 & $3 \times 3$ & 256 & 1 & $32 \times 32 \times 256$ \\
\hline \multicolumn{5}{|l|}{ Bridge } \\
\hline \multicolumn{5}{|l|}{ Residual block 5} \\
\hline Convolutional layer 9 & $3 \times 3$ & 512 & 2 & $16 \times 16 \times 512$ \\
\hline Convolutional layer 10 & $3 \times 3$ & 512 & 1 & $16 \times 16 \times 512$ \\
\hline \multicolumn{5}{|l|}{ Decoder } \\
\hline \multicolumn{5}{|l|}{ Residual block 6} \\
\hline Convolutional layer 11 & $3 \times 3$ & 256 & 1 & $32 \times 32 \times 256$ \\
\hline Convolutional layer 12 & $3 \times 3$ & 256 & 1 & $32 \times 32 \times 256$ \\
\hline \multicolumn{5}{|l|}{ Residual block 7} \\
\hline Convolutional layer 13 & $3 \times 3$ & 128 & 1 & $64 \times 64 \times 128$ \\
\hline Convolutional layer 14 & $3 \times 3$ & 128 & 1 & $64 \times 64 \times 128$ \\
\hline \multicolumn{5}{|l|}{ Residual block 8} \\
\hline Convolutional layer 15 & $3 \times 3$ & 64 & 1 & $128 \times 128 \times 64$ \\
\hline Convolutional layer 16 & $3 \times 3$ & 64 & 1 & $128 \times 128 \times 64$ \\
\hline \multicolumn{5}{|l|}{ Residual block 9} \\
\hline Convolutional layer 17 & $3 \times 3$ & 32 & 1 & $256 \times 256 \times 32$ \\
\hline Convolutional layer 18 & $3 \times 3$ & 32 & 1 & $256 \times 256 \times 32$ \\
\hline \multicolumn{5}{|l|}{ Output } \\
\hline \multicolumn{5}{|l|}{ N/A } \\
\hline Convolutional layer 19 & $1 \times 1$ & 1 & 1 & $256 \times 256 \times 1$ \\
\hline \multicolumn{5}{|l|}{ N/A } \\
\hline Sigmoid layer & N/A & N/A & N/A & $256 \times 256 \times 1$ \\
\hline
\end{tabular}

${ }^{\mathrm{a}} \mathrm{N} / \mathrm{A}$ : not applicable; this model part did not include this parameter. 


\section{Implementation}

We implemented our proposed model using the TensorFlow package (version 1.14.0), which provides a Python (version 3.6.8; Python Software Foundation) application programming interface for tensor manipulation. We also used Keras (version 2.2.4) as the official front end of TensorFlow. We trained the models with the Adam optimizer with a learning rate of 0.0001 , a batch size of 16, and the loss functions of binary cross-entropy and dice loss [17] on the GeForce GTX 1080 Ti GPU (NVIDIA Corporation).

For the performance evaluation, 5-fold cross-validation was performed to confirm its generalization ability. The augmented training data set $(\mathrm{n}=48,874)$ was randomly shuffled and divided into five equal groups in a stratified manner. Subsequently, four groups were selected for training the model, and the remaining group was used for validation. This process was repeated five times by shifting the internal validation group. Then, we averaged the mean validation costs of the five internal validation groups according to each epoch and found the optimal epoch that provides the lowest validation cost. The testing data set was evaluated only after the model was completely trained using the training and validation data set.

\section{Performance Evaluation}

We first investigated the effect of the number of residual blocks. For the comparison, we repeated the same procedure of the 5-fold cross-validation for two to five residual blocks. For further performance comparison, we compared our proposed method with U-Net [6], bidirectional U-Net [7], and R2U-Net [8].

For the segmentation evaluation, we quantized the mean intersection over union (mIoU), which is defined as the size of the intersection divided by the size of the union. Particularly for the nonascites images, no pixel was segmented, as we quantized the value by zero. If there were no segmentation results for the nonascites image, we quantized the value by 1 .

In addition to the segmentation performance, we evaluated the detection performance. If the mIoU value was equal or greater than a certain threshold value, we declared it by ascites image. For the detection performance, we plotted a receiver operating characteristic (ROC) curve and calculated the area under the ROC curve (AUROC). Subsequently, we also evaluated the sensitivity, specificity, accuracy, balanced accuracy, precision, and F1 score. More specifically, we calculated true positives (TPs), false positives (FPs), true negatives (TNs), and false negatives (FNs) and computed the following metrics:

$$
\begin{aligned}
& \text { Sensitivity }=\mathrm{TP} /(\mathrm{TP}+\mathrm{FN})(\mathbf{1}) \\
& \text { Specificity }=\mathrm{TN} /(\mathrm{TN}+\mathrm{FP})(\mathbf{2}) \\
& \text { Accuracy }=(\mathrm{TP}+\mathrm{TN}) /(\mathrm{TP}+\mathrm{TN}+\mathrm{FP}+\mathrm{FN})(\mathbf{3}) \\
& \text { Balanced Accuracy }=(\text { Sensitivity }+ \text { Specificity }) / 2 \\
& (\mathbf{4}) \\
& \text { Precision }=\mathrm{TP} /(\mathrm{TP}+\mathrm{FP})(\mathbf{5}) \\
& \text { F1 score }=2 \times(\text { Sensitivity } \times \text { Precision }) /(\text { Sensitivity } \\
& + \text { Precision })(\mathbf{6})
\end{aligned}
$$

where TP is the amount of ascites data correctly classified as ascites, TN is the amount of nonascites data correctly classified as normal, FP is the amount of nonascites data misclassified as ascites, and FN is the amount of ascites data misclassified as normal. Two abdominal radiologists ( $\mathrm{JH}$ and $\mathrm{KWK}$ ) also evaluated the factors influencing the performance of detection and segmentation of ascites through a systematic review of all original CT images and AI results of the testing data set.

\section{Results}

\section{Performance in the Cross-Validation}

Table 4 summarizes the cross-validation results of various AI models for ascites segmentation performance and ascites detection accuracy using mIoU and AUROC, respectively. Deep residual U-Net models with various numbers of residual blocks generally provided higher mIoU and AUROC values than any other state-of-the-art methods [6-8]. Among the deep residual U-Net models with various numbers of residual blocks, the model with four residual blocks provided the highest mIoU (0.87) for the segmentation performance and the highest AUROC (0.99) for the detection performance. The computational time for training for the deep residual U-Net model with four residual blocks and 5-fold cross-validation was 27 hours. The overall computational time for testing was 30 minutes.

Table 4. Cross-validation results for the training data set comparing the mIoU for segmentation performance and AUROC for detection across models.

\begin{tabular}{lll}
\hline Model & $\operatorname{mIoU}^{\mathrm{a}}(\mathrm{SD})$ & $\mathrm{AUROC}^{\mathrm{b}}(\mathrm{SD})$ \\
\hline Deep residual U-Net (two residual blocks) & $0.86(0.03)$ & $0.97(0.02)$ \\
Deep residual U-Net (three residual blocks) & $0.86(0.02)$ & $0.98(0.01)$ \\
Deep residual U-Net (four residual blocks) & $0.87(0.02)$ & $0.99(0.01)$ \\
Deep residual U-Net (five residual blocks) & $0.69(0.46)$ & $0.69(0.01)$ \\
U-Net [6] & $0.84(0.02)$ & $0.96(0.01)$ \\
Bidirectional U-Net [7] & $0.82(0.01)$ & $0.91(0.01)$ \\
Recurrent residual U-Net [8] & $0.74(0.02)$ & $0.90(0.01)$ \\
\hline
\end{tabular}

amIoU: mean intersection over union; this is an index of the segmentation performance.

${ }^{\mathrm{b}}$ AUROC: area under the receiver operating characteristic curve; this is an index of detection accuracy. 
We also investigated the effect of the number of convolutional layers in each residual block. Table 5 summarizes the cross-validation results when the number of convolutional layers changes from two to four. It shows that the deep residual U-Net model with the two convolutional layers in each residual block provided the highest values of mIoU (0.87) and AUROC (0.99), followed by three convolutional layers (mIoU $=0.83$ and AUROC $=0.98$ ) and four convolutional layers (mIoU $=0.69$ and AUROC $=0.69$ ).

Table 5. Effect of the number of convolutional layers in each residual block on cross-validation results with the training data set.

\begin{tabular}{|c|c|c|}
\hline Model & $\mathrm{mIoU}^{\mathrm{a}}(\mathrm{SD})$ & $\mathrm{AUROC}^{\mathrm{b}}(\mathrm{SD})$ \\
\hline Deep residual U-Net with two convolutional layers in each residual block & $0.87(0.02)$ & $0.99(0.01)$ \\
\hline Deep residual U-Net with four convolutional layers in each residual block & $0.69(0.02)$ & $0.69(0.01)$ \\
\hline
\end{tabular}

${ }^{a}$ mIoU: mean intersection over union; this is an index of the segmentation performance.

${ }^{b}$ AUROC: area under the receiver operating characteristic curve; this is an index of the detection accuracy.

\section{Performance With the Testing Data Set}

Table 6 summarizes the testing data results for segmentation performance using mIoU and detection accuracy using AUROC when the number of convolutional layers changes from two to four. Similar to the cross-validation results, these results also show that the deep residual U-Net model with four residual blocks including two convolutional layers provided the highest mIoU (0.87) and AUROC (0.96) with the isolated testing data set $(n=1635)$.

With the two convolutional layers in each residual block, we also evaluated and compared the segmentation and detection performances. For the performance comparison, we changed the number of residual blocks from two to five and tested each model using the testing data set. Also, we tested with U-Net, bidirectional U-Net, and R2U-Net. Table 7 summarizes the performance comparison. The results also show that the deep residual U-Net with four residual blocks provided the highest mIoU and AUROC values. We also note that the deep residual U-Net with three residual blocks also provided high values of mIoU and AUROC, which were higher than any other state-of-the-art methods, indicating that the deep residual U-Net approach was more appropriate for the ascites segmentation and detection.

The representative images of ascites segmentation are presented in Figure 2. The left-hand column (A) includes the original CT images and the ground-truth masking images. Five examples of ascites segmentation results are shown using our proposed model (B) and comparing them with those using U-Net (C), bidirectional U-Net (D), and R2U-Net (E). Our proposed model correctly segmented the ascites region regardless of its pattern and size (the top four panels in column B). In addition, for the nonascites images, the segmentation results were not shown (the bottom panel in column B).

Table 8 summarizes the testing data results of detection accuracy with the metrics of sensitivity, specificity, accuracy, balanced accuracy, precision, and F1 score. The deep residual U-Net with four residual blocks provided the highest accuracy metrics: sensitivity $=0.96$, specificity $=0.96$, accuracy $=0.96$, balanced accuracy $=0.96$, precision $=0.91$, and F1 score $=0.93$. Based on these results, we proposed our deep residual U-Net with four residual blocks as an optimal AI algorithm for automatic ascites detection and segmentation on abdominopelvic CT scans.

Table 6. Effect of the number of convolutional layers in each residual block on the testing data set results for the deep residual U-Net model with four residual blocks.

\begin{tabular}{lll}
\hline Model & mIoU $^{\mathrm{a}}$ (SD) & AUROC $^{\mathrm{b}}$ \\
\hline Deep residual U-Net with two convolutional layers in each residual block & $0.87(0.26)$ & 0.96 \\
Deep residual U-Net with three convolutional layers in each residual block & $0.84(0.27)$ & 0.94 \\
Deep residual U-Net with four convolutional layers in each residual block & $0.74(0.31)$ & 0.72 \\
\hline
\end{tabular}

amIoU: mean intersection over union; this is an index of the segmentation performance.

${ }^{b}$ AUROC: area under the receiver operating characteristic curve; this is an index of the detection accuracy. 
Table 7. Segmentation performance and detection accuracy of artificial intelligence models with the testing data set.

\begin{tabular}{lll}
\hline Model & $\operatorname{mIoU}^{\mathrm{a}}(\mathrm{SD})$ & AUROC $^{\mathrm{b}}$ \\
\hline Deep residual U-Net (two residual blocks) & $0.81(0.33)$ & 0.87 \\
Deep residual U-Net (three residual blocks) & $0.86(0.28)$ & 0.93 \\
Deep residual U-Net (four residual blocks) & $0.87(0.26)$ & 0.96 \\
Deep residual U-Net (five residual blocks) & $0.70(0.46)$ & 0.70 \\
U-Net [6] & $0.80(0.33)$ & 0.90 \\
Bidirectional U-Net [7] & $0.77(0.35)$ & 0.86 \\
Recurrent residual U-Net [8] & $0.67(0.41)$ & 0.81 \\
\hline
\end{tabular}

${ }^{a}$ mIoU: mean intersection over union; this is an index of the segmentation performance.

${ }^{b}$ AUROC: area under the receiver operating characteristic curve; this is an index of the detection accuracy.

Figure 2. Five examples of ascites segmentation results using each model. A. The original computed tomography (CT) images and the ground-truth masking images. B. Our proposed model. C. The U-Net model. D. The bidirectional U-Net model. E. The recurrent residual U-Net model. Each row represents a different example of CT images. Blue represents the ground-truth masking images, and red represents the resultant segmented images.
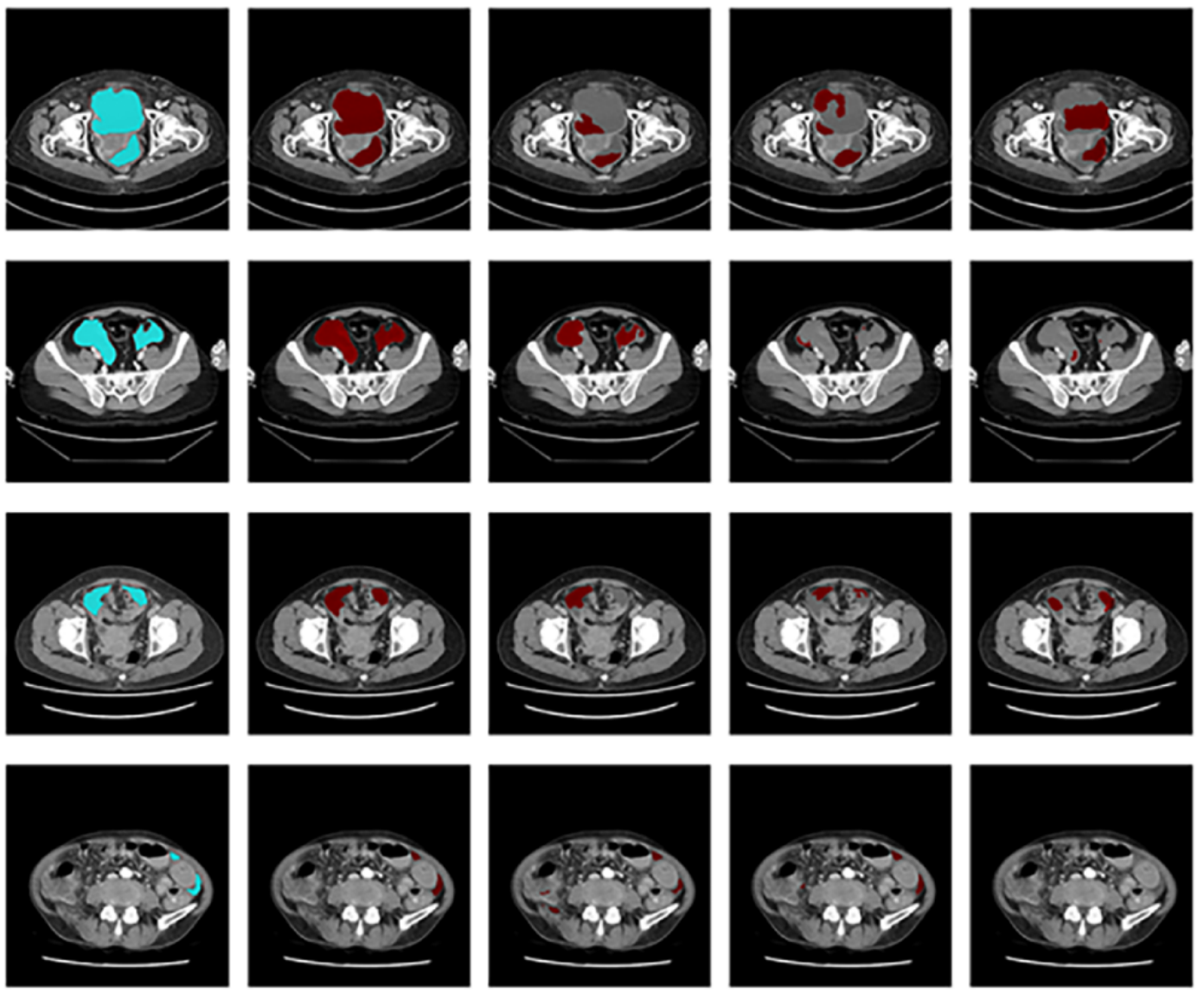

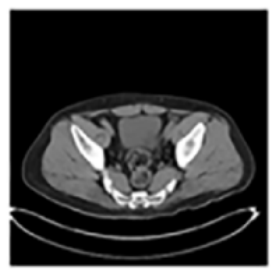

A

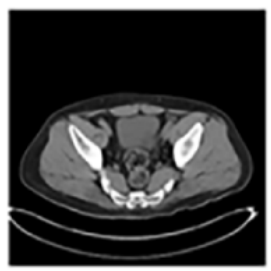

B

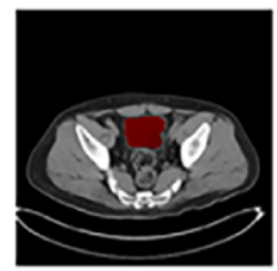

C

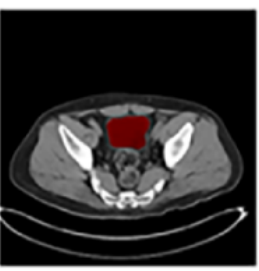

$\mathrm{D}$

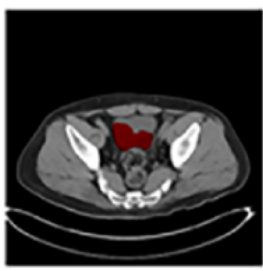

E 
Table 8. Detection performance metrics of artificial intelligence models with the testing data set.

\begin{tabular}{lllllll}
\hline Model & Sensitivity & Specificity & Accuracy & Balanced accuracy & Precision & F1 score \\
\hline U-Net [6] & 0.92 & 0.90 & 0.90 & 0.91 & 0.79 & 0.85 \\
Bidirectional U-Net [7] & 0.94 & 0.86 & 0.88 & 0.90 & 0.74 & 0.83 \\
Recurrent residual U-Net [8] & 0.85 & 0.81 & 0.82 & 0.83 & 0.66 & 0.74 \\
$\begin{array}{l}\text { Deep residual U-Net } \\
\text { (four residual blocks) }\end{array}$ & 0.96 & 0.96 & 0.96 & 0.96 & 0.91 & 0.93 \\
\hline
\end{tabular}

\section{Factors Influencing the Performance}

Through the expert review of all images in the testing data set by two radiologists ( $\mathrm{JH}$ and $\mathrm{KWK}$ ), there were two categories of false positive images. The AI algorithm could not differentiate between ovarian cysts of a substantial size $(>3 \mathrm{~cm}$ in diameter) and ascites (Figure 3A). In contrast, normal physiologic ovarian cysts were correctly identified by our algorithm. The AI algorithm could not differentiate ascites from a fully distended urinary bladder (Figure 3B). However, the AI algorithm was able to differentiate ascites from a partially distended or collapsed urinary bladder.

All the false negative images showed a small amount of ascites. Two radiologists determined that all the false negative results were clinically insignificant.

Figure 3. Examples of incorrect segmentation results. The left-hand column includes the original computed tomography (CT) images, the middle column includes the ground-truth masking images, and the right-hand column includes the segmented results by our deep residual U-Net algorithm. A. In a patient with a left ovarian cyst, our artificial intelligence (AI) algorithm detected fluid within the ovarian cyst as ascites. B. In a patient with a fully distended bladder, our AI algorithm detected fluid in the bladder as ascites. Red represents the resultant segmented images.

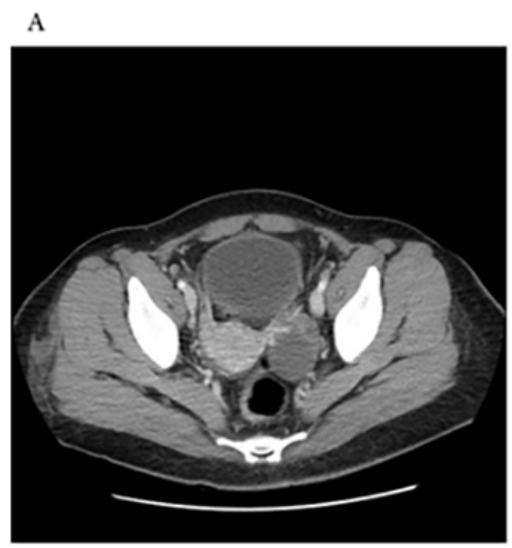

B

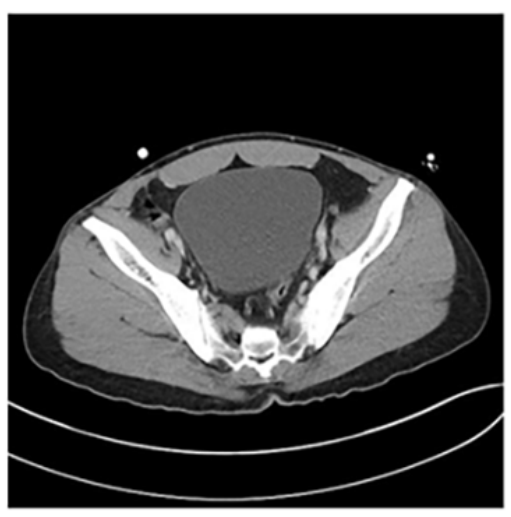

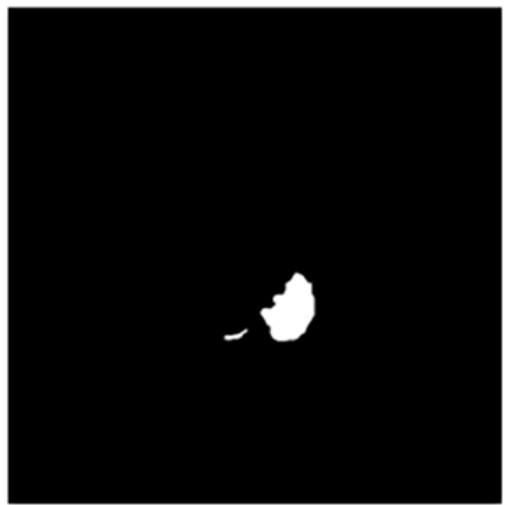

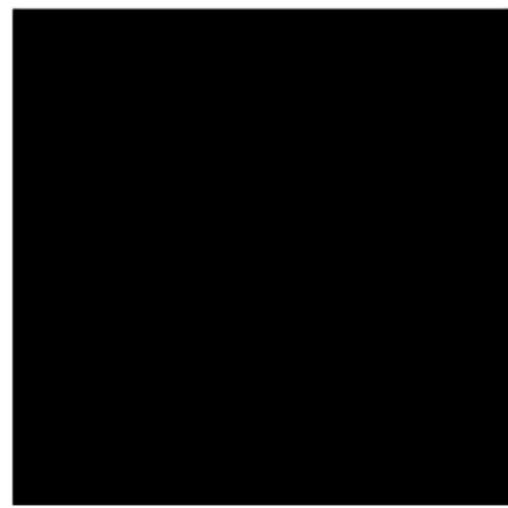

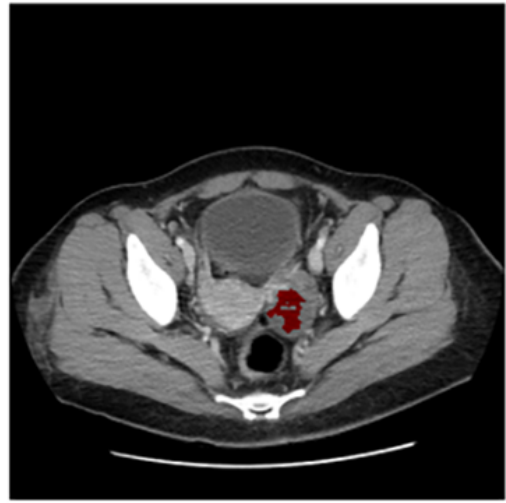

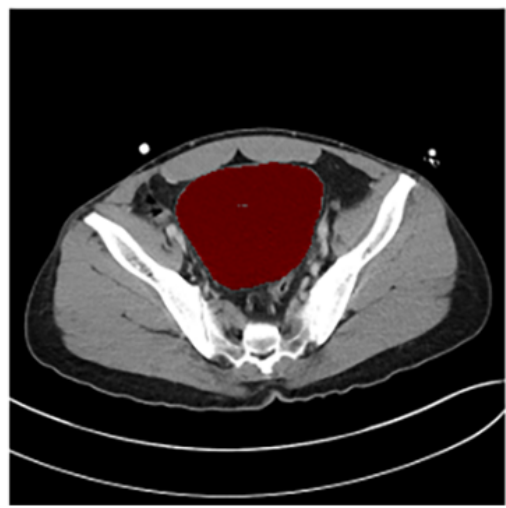

\section{Discussion}

\section{Principal Findings}

In this study, for the first time, we developed a deep residual U-Net model for the segmentation of ascites on CT images, which provided higher accuracy compared with state-of-the-art networks, including U-Net, bidirectional U-Net, and R2U-Net. Our study results demonstrated that our AI algorithm was able to detect and quantify ascites in the abdominopelvic cavity. Our proposed algorithm was the deep residual U-Net model, which achieved $96 \%$ sensitivity, $96 \%$ specificity, and $96 \%$ accuracy for ascites detection with the testing data set. The segmentation performance was also high, with an mIoU of 0.87 , when comparing the AI segmentation results and ground-truth values. However, the ground-truth values were generated by a human expert, and human error may have affected the drawing of the ascites boundaries. Thus, we believe that the AI segmentation algorithm might be more accurate for drawing the boundary areas of ascites in general. 
The deep residual U-Net model outperformed the state-of-the-art algorithms, including U-Net, bidirectional U-Net, and R2U-Net. The deep residual U-Net model combined the strengths of residual learning and U-Net architecture [9]. The network was built with residual units and has similar architecture to that of U-Net. The benefits of this model are three-fold: (1) residual units facilitate the training of deep networks, (2) the vanishing gradient problem is reduced, and (3) the rich skip connections within the network could facilitate information propagation, resulting in higher mIoU values. Integration of the residual network with standard U-Net architecture enabled us to extract robust discriminative features from input CT images.

In general, the concept of U-Net is to stitch low-level features into corresponding high-level features, thereby adding low-level texture features to high-level semantic features. Thus, U-Net with a deep layer can provide better segmentation results.

Table 9. Comparison of the number of parameters for each U-Net model.

\begin{tabular}{llll}
\hline Model & Trainable parameters, $\mathrm{n}$ & Nontrainable parameters, $\mathrm{n}$ & Total parameters, $\mathrm{n}$ \\
\hline Our proposed model & $18,840,545$ & 14,592 & $18,855,137$ \\
U-Net [6] & $34,600,353$ & 14,016 & $34,614,369$ \\
Bidirectional U-Net [7] & $55,398,798$ & 1408 & $55,400,197$ \\
Recurrent residual U-net [8] & $24,133,013$ & 0 & $24,133,013$ \\
\hline
\end{tabular}

So far, there has been only one study that developed an AI algorithm to detect ascites [3]. In that study, the authors used a CNN algorithm mainly for the classification of three abnormal CT findings of free fluid (ie, ascites), free gas, and mesenteric fat stranding. The accuracy of the CNN algorithm achieved $85 \%$ sensitivity and $95 \%$ specificity to detect ascites. In contrast, our deep residual U-Net algorithm achieved $96 \%$ sensitivity and $96 \%$ specificity for ascites detection. In addition, our deep residual U-Net algorithm also quantified the amount of ascites with high segmentation accuracy (mIoU $=0.87$ ). Thus, we believe that it is quite possible to use our proposed algorithm for ascites detection and quantification on abdominopelvic CT images in patients who visit the emergency department.

In the majority of urgent and emergent situations, clinicians should read the CT scan without radiologic support immediately after the CT scan was obtained. Getting a radiology report usually takes time, and radiologic support may not be maintained 24 hours per day in many institutions [20]. AI algorithms can help maintain radiology support in real time with high diagnostic accuracy. Our training and test data sets were unique in that CT data were obtained from patients who visited the emergency department of a tertiary care hospital, which is designated as a regional emergency medical center and a regional trauma center in Korea. Currently, we incorporated our deep residual U-Net algorithm in our radiology unit and will start further training of our algorithm in a sustainable manner.

There were false positive cases in which our AI algorithm identified fluid within organs, such as the bladder and ovarian cysts, as ascites (Figure 3). These false positive cases will decrease as we continue to train the AI algorithm. All the false
However, an excessive increase in the number of network layers tends to decrease segmentation accuracy. This issue can be solved by adding a residual unit to U-Net, which can make use of the merits of the residual network [6]. A deep residual U-Net model has been used for lung segmentation in CT scans [9], joint segmentation in CT scans [18], and vulnerable plaque segmentation in optical coherence tomography images [19]. These prior studies consistently reported the high segmentation performance of a deep residual U-Net model. In addition, our proposed deep residual U-Net model has an advantage over other U-Net models, in that it requires fewer parameters compared to other tree models [6-8]. Table 9 summarizes the comparison of the number of parameters for each model. Our proposed model includes $18,855,137$ weights and biases, which represents only $54.5 \%$ of the parameters from U-Net. Also, this represents only $34.0 \%$ and $78.1 \%$ of the parameters from bidirectional U-Net and R2U-Net, respectively.

negative cases showed a small amount of ascites, especially between internal organs, such as the bowels, bladder, and uterus. Further training will increase the sensitivity of the AI algorithm to detect ascites.

We adopted a 2D AI algorithm for sequential 2D image analyses rather than a 3D framework, because 3D deep learning requires higher computational power than 2D deep learning [20]. In an emergent clinical setting, a rapid AI algorithm may be preferable to a complex and slow algorithm. Our study showed that sequential 2D image analyses could provide excellent diagnostic accuracy for detecting and quantifying ascites.

\section{Limitations and Future Work}

Our study has several limitations. Firstly, we trained our model using a relatively small amount of CT data. Thus, we will establish a sustainable AI training system and train our AI algorithm using real-world CT data prospectively obtained from our emergency department. Secondly, our AI model was validated internally using a split testing data set. The testing data set was obtained from the same source as the training data set. This may raise issues of generalizability and overfitting of our model [21]. Thus, in the near future, we will validate our model using data from various institutions.

\section{Conclusions}

We propose our deep residual U-Net algorithm for the automatic detection and quantification of ascites in abdominopelvic CT scans. Our model outperformed other state-of-the-art segmentation algorithms based on U-Net, bidirectional U-Net, and R2U-Net. 


\section{Acknowledgments}

This work was supported by the National Research Foundation of Korea (grant 2019R1I1A1A01060744), a grant from the Korea Health Industry Development Institute (grant HI18C1216), and the Korea Medical Device Development Fund grant, which is funded by the Government of the Republic of Korea (the Ministry of Science and ICT; the Ministry of Trade, Industry and Energy; the Ministry of Health and Welfare; and the Ministry of Food and Drug Safety) (grant KMDF_PR_20200901_0095).

\section{Authors' Contributions}

HK and HC carried out the machine learning and deep learning simulation for the hyperparameter search and modeling. JH, JKK, and JL provided the data and performed the data validation to be applied to ascites segmentation. KWK and YK validated and confirmed the simulations and helped to draft the manuscript. HK, JH, and JL wrote the initial manuscript. JL, JH, and KWK conceived the study and participated in its design and coordination. All authors read and approved the final manuscript.

\section{Conflicts of Interest}

None declared.

\section{References}

1. Levine CD, Patel UJ, Wachsberg RH, Simmons MZ, Baker SR, Cho KC. CT in patients with blunt abdominal trauma: Clinical significance of intraperitoneal fluid detected on a scan with otherwise normal findings. AJR Am J Roentgenol 1995 Jun;164(6):1381-1385. [doi: 10.2214/ajr.164.6.7754877] [Medline: 7754877]

2. Wang DC, Parry CR, Feldman M, Tomlinson G, Sarrazin J, Glanc P. Acute abdomen in the emergency department: Is CT a time-limiting factor? AJR Am J Roentgenol 2015 Dec;205(6):1222-1229. [doi: 10.2214/AJR.14.14057] [Medline: 26587929]

3. Winkel DJ, Heye T, Weikert TJ, Boll DT, Stieltjes B. Evaluation of an AI-based detection software for acute findings in abdominal computed tomography scans. Invest Radiol 2019;54(1):55-59. [doi: 10.1097/rli.0000000000000509]

4. Urban BA, Fishman EK. Tailored helical CT evaluation of acute abdomen. Radiographics 2000;20(3):725-749. [doi: 10.1148/radiographics.20.3.g00ma12725] [Medline: 10835125]

5. Sirlin CB, Casola G, Brown MA, Patel N, Bendavid EJ, Hoyt DB. Quantification of fluid on screening ultrasonography for blunt abdominal trauma: A simple scoring system to predict severity of injury. J Ultrasound Med 2001 Apr;20(4):359-364. [doi: 10.7863/jum.2001.20.4.359] [Medline: 11316314]

6. Ronneberger O, Fischer P, Brox T. U-Net: Convolutional networks for biomedical image segmentation. In: Proceedings of the 18th International Conference on Medical Image Computing and Computer-Assisted Intervention. 2015 Presented at: 18th International Conference on Medical Image Computing and Computer-Assisted Intervention; October 5-9, 2015; Munich, Germany p. 234-241. [doi: 10.1007/978-3-319-24574-4 28]

7. Azad TD, Asadi-Aghbolaghi M, Fathy M, Escalera S. Bi-directional ConvLSTM U-Net with densely connected convolutions. In: Proceedings of the IEEE/CVF International Conference on Computer Vision Workshop. 2019 Presented at: IEEE/CVF International Conference on Computer Vision Workshop; October 27-28, 2019; Seoul, Korea p. 406-415. [doi: 10.1109/ICCVW.2019.00052]

8. Alom MZ, Yakopcic C, Hasan M, Taha TM, Asari VK. Recurrent residual U-Net for medical image segmentation. J Med Imaging 2019 Jan 1;6(01):1. [doi: 10.1117/1.jmi.6.1.014006]

9. Khanna A, Londhe N, Gupta S, Semwal A. A deep residual U-Net convolutional neural network for automated lung segmentation in computed tomography images. Biocybern Biomed Eng 2020 Jul;40(3):1314-1327. [doi: 10.1016/j.bbe.2020.07.007]

10. Saeedizadeh N, Minaee S, Kafieh R, Yazdani S, Sonka M. COVID TV-Unet: Segmenting COVID-19 chest CT images using connectivity imposed Unet. Comput Methods Programs Biomed Update 2021;1:100007 [FREE Full text] [doi: 10.1016/j.cmpbup.2021.100007] [Medline: 34337587]

11. Schreier J, Attanasi F, Laaksonen H. A full-image deep segmenter for CT images in breast cancer radiotherapy treatment. Front Oncol 2019;9:677 [FREE Full text] [doi: 10.3389/fonc.2019.00677] [Medline: 31403032]

12. Dong X, Lei Y, Wang T, Thomas M, Tang L, Curran WJ, et al. Automatic multiorgan segmentation in thorax CT images using U-net-GAN. Med Phys 2019 May;46(5):2157-2168 [FREE Full text] [doi: 10.1002/mp.13458] [Medline: 30810231]

13. Ma X, Hadjiiski LM, Wei J, Chan H, Cha KH, Cohan RH, et al. U-Net based deep learning bladder segmentation in CT urography. Med Phys 2019 Apr;46(4):1752-1765 [FREE Full text] [doi: 10.1002/mp.13438] [Medline: 30734932]

14. Amer A, Ye X, Janan F. ResDUnet: A deep learning-based left ventricle segmentation method for echocardiography. IEEE Access 2021;9:159755-159763. [doi: 10.1109/access.2021.3122256]

15. Singadkar G, Mahajan A, Thakur M, Talbar S. Deep deconvolutional residual network based automatic lung nodule segmentation. J Digit Imaging 2020 Jun;33(3):678-684 [FREE Full text] [doi: 10.1007/s10278-019-00301-4] [Medline: $\underline{32026218}]$

16. Mique Jr E, Malicdem A. Deep residual U-Net based lung image segmentation for lung disease detection. In: Proceedings of the International Conference on Information Technology and Digital Applications. 2020 Presented at: International 
Conference on Information Technology and Digital Applications; November 15, 2019; Yogyakarta, Indonesia URL: https:/ /iopscience.iop.org/article/10.1088/1757-899X/803/1/012004/pdf [doi: 10.1088/1757-899X/803/1/012004]

17. Jadon S. A survey of loss functions for semantic segmentation. In: Proceedings of the IEEE Symposium on Computational Intelligence and Bioinformatics and Computational Biology. 2020 Presented at: IEEE Symposium on Computational Intelligence and Bioinformatics and Computational Biology; October 27-29, 2020; Via del Mar, Chile p. 1-7. [doi: $10.1109 /$ cibcb48159.2020.9277638]

18. Rassadin A. Deep residual 3D U-Net for joint segmentation and texture classification of nodules in lung. In: Proceedings of the 17th International Conference on Image Analysis and Recognition. 2020 Presented at: 17th International Conference on Image Analysis and Recognition; June 24-26, 2020; Póvoa de Varzim, Portugal p. 419-427. [doi: 10.1007/978-3-030-50516-5_37]

19. Li L, Jia T. Optical coherence tomography vulnerable plaque segmentation based on deep residual U-Net. Rev Cardiovasc Med 2019 Sep 30;20(3):171-177 [FREE Full text] [doi: 10.31083/j.rcm.2019.03.5201] [Medline: 31601091]

20. Ko H, Chung H, Kang WS, Kim KW, Shin Y, Kang SJ, et al. COVID-19 pneumonia diagnosis using a simple 2D deep learning framework with a single chest CT image: Model development and validation. J Med Internet Res 2020 Jun 29;22(6):e19569 [FREE Full text] [doi: 10.2196/19569] [Medline: 32568730]

21. Kim DW, Jang HY, Kim KW, Shin Y, Park SH. Design characteristics of studies reporting the performance of artificial intelligence algorithms for diagnostic analysis of medical images: Results from recently published papers. Korean J Radiol 2019 Mar;20(3):405-410 [FREE Full text] [doi: 10.3348/kjr.2019.0025] [Medline: 30799571]

\author{
Abbreviations \\ AI: artificial intelligence \\ AUROC: area under the receiver operating characteristic curve \\ CNN: convolutional neural network \\ CT: computed tomography \\ DICOM: Digital Imaging and Communications in Medicine \\ DLM: deep learning model \\ FN: false negative \\ FP: false positive \\ HU: Hounsfield Unit \\ mIoU: mean intersection over union \\ R2U-Net: recurrent residual U-Net \\ ROC: receiver operating characteristic \\ TN: true negative \\ TP: true positive
}

\author{
Edited by G Eysenbach; submitted 22.10.21; peer-reviewed by C Jeong, T Zhang; comments to author 15.11.21; revised version \\ received 30.11.21; accepted 30.11.21; published 03.01.22 \\ Please cite as: \\ Ko H, Huh J, Kim KW, Chung H, Ko Y, Kim JK, Lee JH, Lee J \\ A Deep Residual U-Net Algorithm for Automatic Detection and Quantification of Ascites on Abdominopelvic Computed Tomography \\ Images Acquired in the Emergency Department: Model Development and Validation \\ J Med Internet Res 2022;24(1):e34415 \\ URL: https://www.jmir.org/2022/1/e34415 \\ doi: $10.2196 / 34415$ \\ PMID:
}

CHoon Ko, Jimi Huh, Kyung Won Kim, Heewon Chung, Yousun Ko, Jai Keun Kim, Jei Hee Lee, Jinseok Lee. Originally published in the Journal of Medical Internet Research (https://www.jmir.org), 03.01.2022. This is an open-access article distributed under the terms of the Creative Commons Attribution License (https://creativecommons.org/licenses/by/4.0/), which permits unrestricted use, distribution, and reproduction in any medium, provided the original work, first published in the Journal of Medical Internet Research, is properly cited. The complete bibliographic information, a link to the original publication on https://www.jmir.org/, as well as this copyright and license information must be included. 